\title{
Structural insights into NHEJ: building up an integrated picture of the dynamic DSB repair super complex.
}

\author{
Michal Hammel mhammel@|bl.gov
}

\author{
Molecular Biophysics and Integrated Bioimaging, Lawrence Berkeley National Laboratory, \\ Berkeley, CA 94720, USA.
}

Non-homologous end joining (NHEJ) is the major pathway for repair of DNA double-strand breaks (DSBs) in human cells. NHEJ acts in the generation and prevention of non-homologous chromosomal translocations, a hallmark of genomic instability and many human cancers. X-ray crystal structures, cryoelectron microscopy envelopes, and small angle X-ray scattering (SAXS) solution conformations and assemblies are defining most of the core protein components for NHEJ: Ku70/Ku80 heterodimer; the DNA dependent protein kinase catalytic subunit (DNA-PKcs); the structure-specific endonuclease Artemis along with polynucleotide kinase/phosphatase (PNKP), aprataxin and PNKP related protein (APLF); the scaffolding proteins XRCC4 and XLF (XRCC4-like factor); DNA polymerases, and DNA ligase IV (Lig IV). The dynamic assembly of multi-protein NHEJ complexes at DSBs is regulated in part by protein phosphorylation. The basic steps of NHEJ have been biochemically defined to require: (1) DSB detection by the Ku heterodimer with subsequent DNA-PKcs tethering to form the DNA-PKcs-Ku-DNA complex (termed DNA-PK), (2) lesion processing, and (3) DNA end ligation by Lig IV, which functions in complex with XRCC4 and XLF. The current integration of structures by combined methods is resolving puzzles regarding the mechanisms, coordination and regulation of these three basic steps. Overall, structural results suggest the NHEJ system forms a flexing scaffold with the DNA-PKcs HEAT repeats acting as compressible macromolecular springs suitable to store and release conformational energy to apply forces to regulate NHEJ complexes and the DNA substrate for DNA end protection, processing, and ligation. 